Reprod. Nutr. Dévelop., 1982, 22 (4), 597-610.

\title{
Etude des réponses motrices de l'utérus aux stimulations vaginales et utérines chez la Brebis en cstrus
}

\author{
Marie-Jeanne PRUD'HOMME, J.-P. ROUSSEAU (*) \\ I.N.R.A., Station de Physiologie de la Reproduction, Nouzilly, 37380 Monnaie, France. \\ (*) Université des Sciences et techniques de Lille, Laboratoire de Neurophysiologie végétative \\ (LA 308 - CNRS), Bât. SN4 - 59655 Villeneuve d'Ascq Cedex.
}

Summary. Study of uterine motor responses to vaginal and uterine stimulations in the ewe in oestrus.

We recorded in vivo the electromyographic (EMG) activity of the myometrium in 36 conscious ewes, including ovariectomized animals treated with oestrogen and cyclic animals in oestrus. We studied the motor responses of the uterus to distension of the vagina with a speculum and distension of the uterine horn with a balloon. These two types of stimulation provoked an increase in myometrial activity. By comparing the responses before and after hypophysectomy and before and after treatment with an $\alpha-$ adrenergic blocking agent, we were able to evaluate the nature of these responses.

Vaginal stimulation induced two types of response :

1) a response of long duration, possibly the result of a spinal reflex followed by a reflex release of oxytocin ;

2) a response of short duration, probably resulting simply from a spinal reflex whose efferent pathway is composed of sympathetic fibres.

Uterine stimulation activated motility in the stimulated horn only. Thus, the observed increase in EMG activity was not a reflex but the response of the uterine muscle to its own distension.

\section{Introduction.}

Les distensions du vagin, du cervix et de l'utérus stimulent la motricité utérine chez la Lapine en post-partum (Ferguson, 1941). L'effet est diminué par l'anesthésie profonde, la lésion de la tige pituitaire, la section de la moelle thoracique. Il résulte d'un réflexe neuro-endocrinien : l'ocytocine serait libérée par la neurohypophyse en réponse à des stimulations portées sur les différents segments du tractus génital femelle. Les résultats ultérieurs de Cross (1958) diffèrent de ceux de Ferguson sur deux points : Cross n'observe pas, chez la lapine castrée, traitée par les œstrogènes et anesthésiée, d'augmentation de la motricité d'une corne utérine à la suite de la dilatation de l'autre corne et du cervix. La dilatation du vagin provoque une augmentation de l'activité utérine, distincte de 
celle produite par l'ocytocine, mais semblable à l'effet de faibles doses d'adrénaline ou de noradrénaline injectées par la voie intraveineuse; la réponse est encore présente après la section de la moelle thoracique ou après l'hypophysectomie, elle est supprimée par l'anesthésie épidurale. La distension vaginale mettrait en jeu un réflexe spinal dont les efférences excitatrices emprunteraient la voie des nerfs sympathiques. Chez la Vache, la Brebis et la Chèvre, la distension vaginale provoque l'éjection du lait (Debackere et Peeters, 1960; Peeters et Debackere, 1963) et chez la Brebis, une augmentation de la pression intramammaire (Richard, 1969). Ces réponses sont vraisemblablement la conséquence de la libération d'ocytocine dont la concentration plasmatique augmente durant la dilatation vaginale chez la Chèvre (Roberts et Share, 1970 ; Blank et De Bias, 1977) et chez la Brebis (Roberts et Share, 1968).

Nous avons recherché les effets de la distension vaginale et utérine sur la motricité utérine chez la Brebis éveillée et la nature des mécanismes qui les soustendent. L'activité électromyographique (EMG) de I'utérus est enregistrée chez des brebis sous imprégnation œstrogénique, activité dont l'évolution est connue tant au cours de l'œstrus induit chez la brebis castrée que de l'œstrus naturel (Rousseau et Prud'homme, 1974). Les réponses du myomètre aux stimulations du tractus génital sont étudiées chez la brebis témoin et comparées à celles observées chez la brebis hypophysectomisée.

\section{Matériel et Méthodes.}

Les expériences sont réalisées sur 36 brebis de race Préalpes, sous imprégnation œstrogénique. 27 brebis sont castrées puis traitées par une injection de $50 \mu \mathrm{g}$ de benzoate d'œstradiol pour déclencher l'œstrus. 9 brebis cycliques sont utilisées au cours de leur cstrus naturel (5) ou d'un cestrus induit (4) par des éponges vaginales imprégnées d'un progestagène (acétate de fluorogestone) avec une supplémentation de PMSG.

Les stimulations du tractus génital et du rectum sont réalisées chez l'animal éveillé au cours de l'œstrus comportemental détecté par le Bélier, soit $24 \mathrm{~h}$ après l'injection d'œstrogènes pour les brebis castrées et traitées, et le jour de l'œstrus pour les brebis cycliques. La dilatation du vagin ou du rectum est effectuée par l'introduction d'un spéculum dont l'écartement des valves est le même $(3 \mathrm{~cm})$ dans toutes les expériences. La corne utérine est distendue par un ballonnet placé à demeure auparavant dans la lumière de la corne et gonflé de $15 \mathrm{ml}$ d'air. Les effets des drogues suivantes sont étudiés le même jour : l'ocytocine (de 5 à $100 \mathrm{mUl}$ ) (Syntocinon-Sandoz), I'adrénaline (25 et $50 \mathrm{\mu g}$ ) (Méram), la noradrénaline $(25$ et $50 \mu \mathrm{g}$ ) (Badrial) et la phentolamine $(5 \mathrm{mg})$ (régitine - Ciba-Geigy). Les doses indiquées sont administrées par voie intraveineuse aux animaux dont le poids moyen est de $50 \mathrm{~kg}$.

L'hypophysectomie est pratiquée par la voie parapharyngée chez 11 des 27 brebis castrées. Après l'intervention, les brebis sont placées en salle conditionnée $\left(24 \pm 2{ }^{\circ} \mathrm{C}\right)$. La déficience des glandes surrénales est palliée par un trai- 
tement par l'hydrocortisone et les animaux peuvent s'abreuver à volonté. Les stimulations du tractus génital, les administrations d'ocytocine et de phentolamine sont réalisées sur les brebis hypophysectomisées au cours d'un œestrus induit par les cestrogènes. Les résultats sont comparés à ceux obtenus sur ces mêmes brebis au cours d'un œstrus induit avant l'hypophysectomie.

L'activité électromyographique (EMG) du myomètre est recueillie en 2 ou 3 points par des électrodes bipolaires de platine placées à demeure selon une technique déjà décrite (Rousseau et Prud'homme, 1974). Les variations de potentiel sont amplifiées par des amplificateurs dont la bande passante est de 0,53 à 150 Hertz. Elles sont enregistrées sur papier grâce à un inscripteur à plumes classique (Beckman).

\section{Résultats.}

I. Effet de la distension du tractus génital et du rectum sur l'activité EMG du myomètre (tabl. 1). Chez les brebis sous imprégnation œstrogénique, l'activité EMG du myomètre est caractérisée par des salves de potentiels, de 6 à $8 \mathrm{~s}$ de durée, contemporaines des contractions de l'utérus. Ces salves se répètent régulièrement au rythme moyen de 2,5/min (Rousseau et Prud'homme, 1974).

\section{TABLEAU 1}

Effet de la distension du tractus génital et du rectum sur l'activité EMG du myomètre.

\begin{tabular}{|c|c|c|c|}
\hline \multirow{2}{*}{$\begin{array}{l}\text { Type de stimulation et } \\
\text { organe stimulé }\end{array}$} & \multirow{2}{*}{$\begin{array}{l}\text { Effet sur l'activité } \\
\text { EMG du myomètre }\end{array}$} & \multicolumn{2}{|c|}{ Caractéristiques de la réponse myométriale } \\
\hline & & Localisation & Durée \\
\hline $\begin{array}{l}\text { Mise en place du spé- } \\
\text { culum dans le vagin }\end{array}$ & + & Les 2 cornes & $\begin{array}{l}\text { dépend de la durée } \\
\text { de la stimulation }\end{array}$ \\
\hline Dilatation vaginale & $0 /+^{*}$ & Les 2 cornes & $\begin{array}{l}\text { toujours supérieure à la } \\
\text { durée de la stimulation }\end{array}$ \\
\hline Distension utérine & + & $\begin{array}{l}\text { La corne stimulée } \\
\text { seulement }\end{array}$ & $\begin{array}{l}\text { égale à la durée de la } \\
\text { stimulation }\end{array}$ \\
\hline
\end{tabular}

Dilatation du rectum

0

* Détails dans le tableau 2.

$1^{\circ}$ Effet de la mise en place du spéculum dans le vagin. - Chez les brebis cycliques en cstrus induit, la seule introduction du spéculum dans le vagin, sans en écarter les valves, provoque une augmentation de l'activité EMG de courte durée. Lorsque l'appareil est laissé en place pendant 30 à $40 \mathrm{~s}$, il est recueilli une bouffée de potentiels, de durée équivalente (fig. 1a, lig. A). Cette salve initiale est suivie d'un rythme de 3 à 4 salves/min, lorsque le spéculum est maintenu plus longtemps dans le vagin (fig. 1a, lig. B). 
$\mathbf{a}$

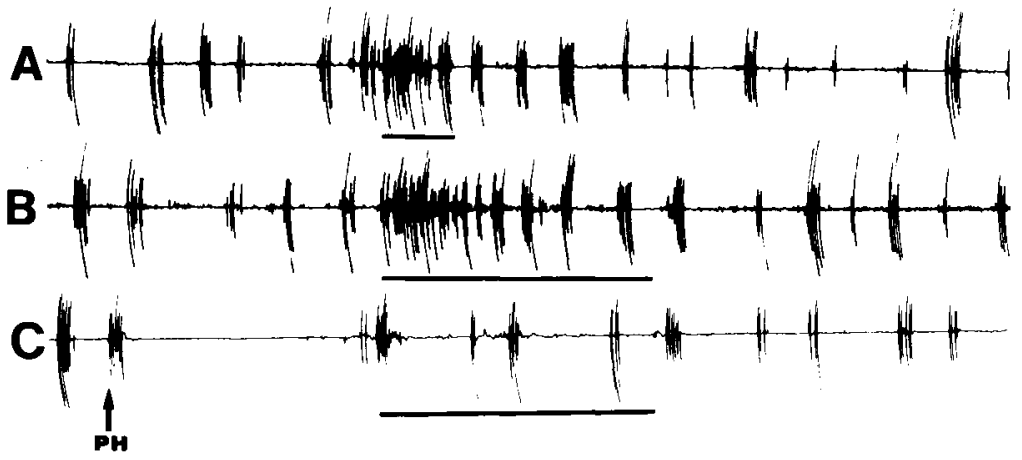

b

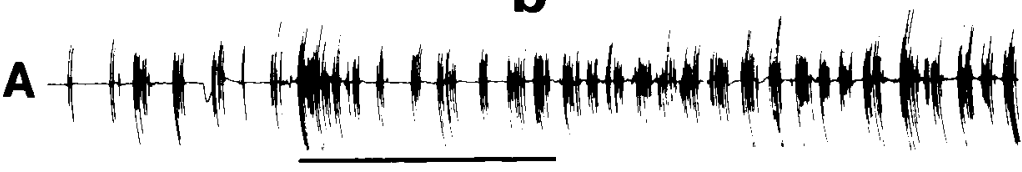

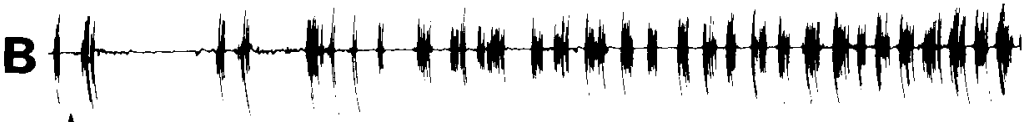

t

C
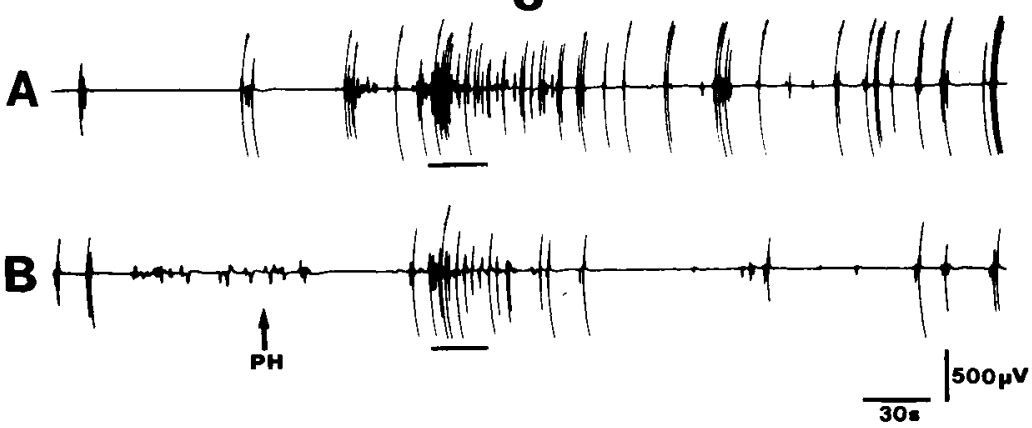

FIG. 1. - Enregistrements de l'activité EMG du myomètre, en un même point d'une corne utérine, dans différentes situations expérimentales. Les traits horizontaux correspondent aux stimulations vaginales :

a) Les enregistrements $A, B$ et $C$ sont obtenus chez une brebis cyclique en ostrus induit. A et B montrent l'augmentation de l'activité EMG en réponse à la seule introduction du spéculum dans le vagin respectivement pendant $30 \mathrm{~s}$ et $2 \mathrm{~min}$. En C, la réponse à une stimulation identique à celle portée en $B$ est nettement diminuée après l'injection de $5 \mathrm{mg}$ de phentolamine ( $\mathrm{PH})$. Seule subsiste une brève salve au tout début de la stimulation. $b$ ) Les enregistrements $A$ et $B$ sont obtenus chez une brebis cyclique en œstrus naturel. La dilatation vaginale induit une réponse de longue durée, caractérisée par une salve initiale de 20 à $30 \mathrm{~s}$, suivie d'une augmentation du rythme (A). En B, l'injection de $5 \mathrm{mg}$ de phentolamine (PH) réduit seulement la durée de la salve initiale. L'augmentation plus tardive du rythme subsiste. c) Les enregistrements $A$ et $B$ sont obtenus chez une brebis castrée traitée par les œstrogènes. Chez cet animal, la dilatation du vagin entraîne une réponse de courte durée (A), réduite après l'injection de phentolamine (PH) en $B$. 
$2^{\circ}$ Effet de la dilatation vaginale. - Nous appelons dilatation vaginale la stimulation induite par la mise en place du spéculum et l'écartement de ses valves.

a) Chez des brebis castrées et traitées par les œestrogènes. - La dilatation du vagin pendant 20 à $30 \mathrm{~s}$, au cours de la saison sexuelle, provoque une augmentation de l'activité utérine sur les deux cornes chez tous les animaux $(n=16)$. La même stimulation pratiquée pendant l'anœstrus saisonnier entraîne une réponse chez seulement 6 des 11 animaux (tabl. 2). La fréquence moyenne des salves s'élève de 2 à 7 par minute dès le début de la dilatation et le maximum est atteint en moins de $30 \mathrm{~s}$; elle évolue selon deux modalités :

- Dans les réponses qualifiées de longue durée, la fréquence de 7 salves/min se maintient pendant 4 à $5 \mathrm{~min}$ et le rythme retourne à sa valeur initiale après un délai d'une quinzaine de minutes (fig. 2A).

- Dans les réponses de courte durée, l'effet ne persiste pas et le rythme des salves revient à la normale dès la deuxième minute, quelle que soit la durée de la stimulation (fig. 2B).

L'amplitude des potentiels ( 1 à $2 \mathrm{mV}$ ) et leur fréquence au sein des salves $(1 / s)$ ne sont pas modifiées. II est recueilli autant de réponses des deux types, quelle que soit la période de l'année où les expériences sont effectuées (tabl. 2).

TABLEAU 2

Répartition des types de réponses du myomètre à la dilatation vaginale.

\begin{tabular}{|c|c|c|c|c|}
\hline \multirow{2}{*}{$\begin{array}{l}\text { Types de réponses } \\
\text { à la dilatation vaginale }\end{array}$} & \multicolumn{2}{|c|}{$\begin{array}{l}\text { Brebis castrée } \\
\text { traitée par les œestrogènes }\end{array}$} & \multicolumn{2}{|c|}{$\begin{array}{c}\text { Brebis cyclique } \\
\text { pendant la saison sexuelle }\end{array}$} \\
\hline & $\begin{array}{c}\text { pendant la } \\
\text { saison sexuelle } \\
\mathbf{n}=16\end{array}$ & $\begin{array}{c}\text { pendant } \\
\text { l'ancestrus } \\
n=11\end{array}$ & $\begin{array}{c}\text { au cours de } \\
\text { l'œestrus naturel } \\
n=5\end{array}$ & $\begin{array}{c}\text { au cours de } \\
\text { l'œstrus induit } \\
n=4\end{array}$ \\
\hline Absence de réponse $\ldots \ldots \ldots$ & 0 & $5(45$ p. 100$)$ & 0 & 0 \\
\hline $\begin{array}{l}\text { Réponse de longue durée } \\
\text { (supérieure à } 15 \mathrm{~min} \text { ) ...... }\end{array}$ & $8(50$ p. 100$)$ & $3(27,5$ p. 100$)$ & 0 & 3 \\
\hline $\begin{array}{l}\text { Réponse de durée intermédiaire } \\
(8-10 \mathrm{~min}) \ldots \ldots \ldots \ldots \ldots \ldots\end{array}$ & 0 & 0 & 5 & 1 \\
\hline $\begin{array}{l}\text { Réponse de courte durée } \\
(2 \mathrm{~min}) \ldots \ldots \ldots \ldots \ldots \ldots\end{array}$ & $8(50$ p. 100$)$ & $3(27,5$ p. 100$)$ & 0 & 0 \\
\hline
\end{tabular}

b) Chez les brebis cycliques en œestrus. - Chez les 5 brebis en œestrus naturel, la dilatation vaginale provoque une augmentation de l'activité EMG du myomètre sur les deux cornes qui dure 8 à $10 \mathrm{~min}$. Dès le début de la stimulation, la fréquence des salves croît de 2 à 7 par minute. Elle chute rapidement dès la fin de la stimulation, pour se stabiliser autour de $4 / \mathrm{min}$, pendant $6 \mathrm{~min}$ (fig. $3 \mathrm{~A}$ ). Cette réponse de durée intermédiaire est également observée chez une des 4 brebis cycliques dont l'œstrus a été induit. Chez les 3 autres, l'augmentation de l'activité utérine persiste pendant 15 à $30 \mathrm{~min}$ et s'apparente plutôt à une réponse de longue durée (fig. $3 \mathrm{~B}$ ). La réponse à la dilatation consiste alors en une bouffée initiale de potentiels, de $30 \mathrm{~s}$ de durée, recueillie dès le début de la 


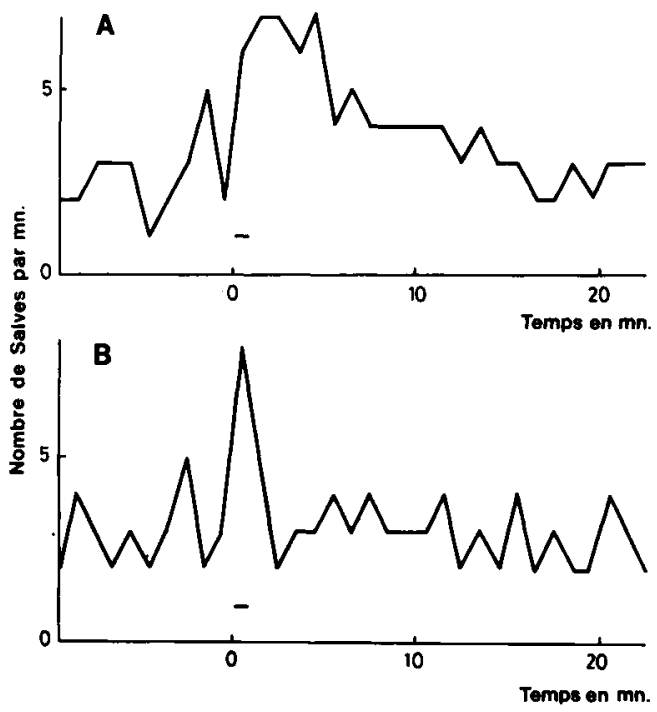

FIG. 2. - Effets de la dilatation vaginale sur la fréquence des salves de potentiels exprimée en nombre par min chez deux brebis castrées traitées par les aestrogènes. Le trait horizontal, placé à partir du temps 0 , marque la durée de la stimulation. La dilatation induit une réponse de longue durée en $A$, de courte durée en $B$.

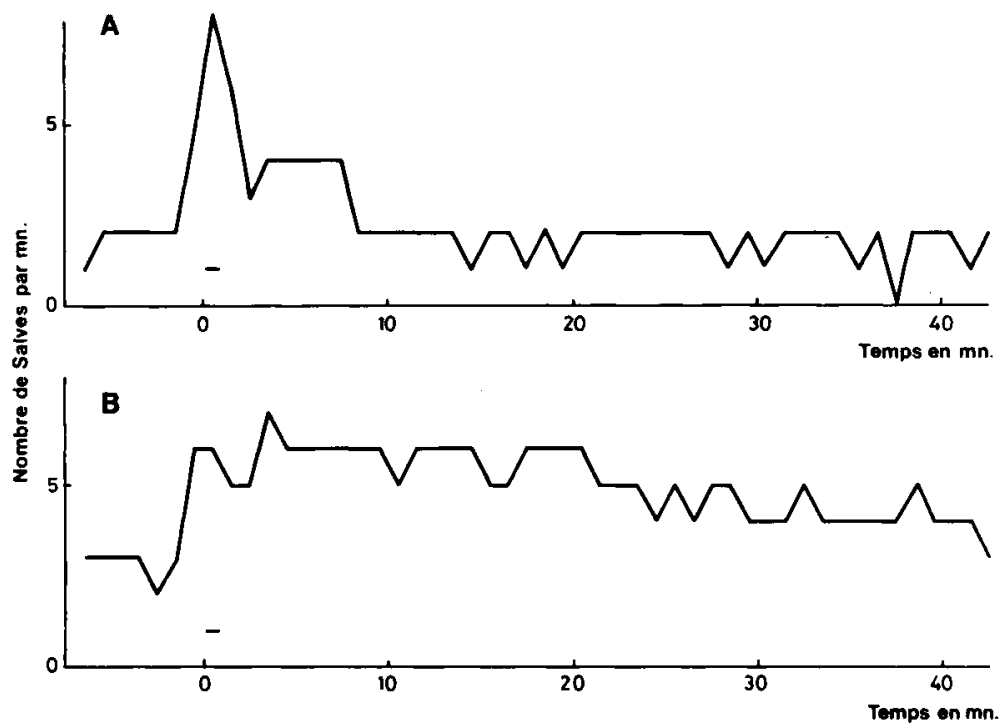

FIG. 3. - Effets de la dilatation vaginale sur la fréquence des salves de potentiels exprimée en nombre par min chez deux brebis cycliques en cestrus. Le trait horizontal à partir du temps 0 marque la durée de la stimulation. La dilatation induit une réponse de durée intermédiaire pendant l'œstrus naturel en $A$, de longue durée pendant l'œestrus induit en $B$. 
distension et suivie d'un rythme transitoire de 4 salves par minute (fig. 1b, lig. A). Deux minutes après le début de la stimulation, la fréquence des salves augmente jusqu'à 6 et 7 par minute. Elle se maintient à ce niveau pendant 5 à 10 min, puis décroît progressivement.

$3^{\circ}$ Effet de la distension utérine. - Chez les brebis castrées et traitées par les cestrogènes la distension de la corne utérine droite provoque une augmentation de la motricité de la seule corne distendue (fig. 4A). L'électromyogramme de la corne stimulée présente plusieurs particularités. Dès le début de la stimulation, l'activité électrique est modifiée de façon caractéristique : pendant 30 à $40 \mathrm{~s}$, la décharge des potentiels apparaît presque continue (effet " on »). Puis, l'activité se présente sous la forme d'une succession de courtes salves dont la fréquence se stabilise autour de $4 / \mathrm{min}$ pendant toute la durée de la distension. L'amplitude des potentiels au sein de ces salves diminue de $400 \mu \mathrm{V}$ à $100 \mu \mathrm{V}$. A l'arrêt de la distension, l'activité enregistrée ressemble à l'effet " on " (effet " off »). Les sal-. ves retrouvent progressivement leur fréquence de base et les potentiels, leur amplitude initiale. La fréquence des salves enregistrées sur la corne contralatérale n'est pas modifiée : elle reste en moyenne de 2 à 3 par minute.
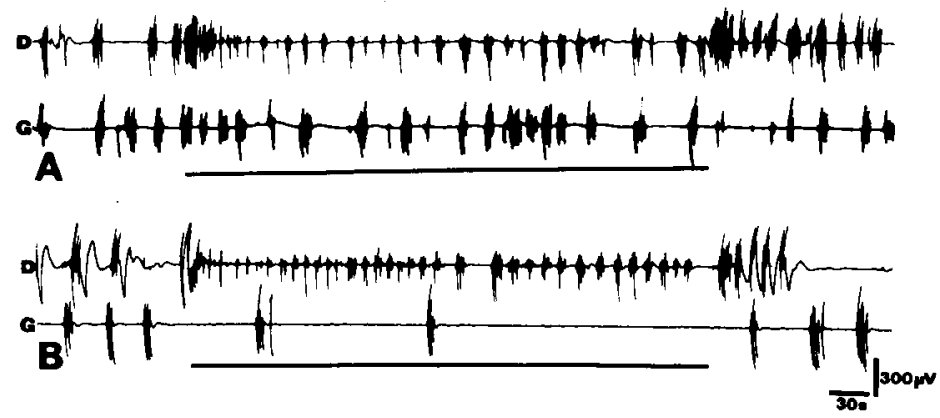

FIG. 4. - Enregistrements de l'activité EMG du myomètre sur les deux cornes, droite (D) et gauche (G), chez une brebis castrée traitée par les œestrogènes. Le trait horizontal correspond à la durée de la distension de la corne droite par un ballonnet. En A, la distension entraîne une augmentation de l'activité EMG de la seule corne stimulée (D). En B, la même stimulation effectuée après hypophysectomie produit un effet identique.

$4^{\circ}$ Effet de la dilatation du rectum. - La dilatation du rectum ne provoque jamais de modification de l'activité utérine.

II. Action de l'ocytocine et des catécholamines sur l'activité EMG du myomètre (tabl. 3). L'injection intraveineuse de $1 \mathrm{ml}$ de sérum physiologique $(\mathrm{NaCl} 0,9 \%)$ n'entraîne aucune modification de l'activité utérine.

$1^{\circ}$ Action de l'ocytocine. - Une dose de $5 \mathrm{mUl} \mathrm{d}^{\prime}$ ocytocine, injectée par voie intraveineuse chez les brebis castrées sous imprégnation œstrogénique, ne modifie pas l'activité utérine. $10 \mathrm{mUl}$ provoquent en $4 \mathrm{~min}$ une augmentation de la fréquence des salves de 2,5 à 5 salves par minute. Cette augmentation dure 4 à $5 \mathrm{~min}$ et l'activité du myomètre retrouve son rythme normal en 8 à 9 min 
TABLEAU 3

Action de l'ocytocine et des catécholamines sur l'activité EMG du myomètre.

\begin{tabular}{|c|c|c|c|}
\hline Substance administrée & $\begin{array}{l}\text { Effet sur I'activité } \\
\text { EMG du myomètre }\end{array}$ & Réponse bilatérale & $\begin{array}{l}\text { Durée de la } \\
\text { réponse }\end{array}$ \\
\hline Sérum Physiologique & 0 & & \\
\hline \multicolumn{4}{|l|}{ Ocvtocine } \\
\hline \multicolumn{4}{|l|}{$\begin{array}{l}\text { - brebis castrée traitée par les } \\
\text { œstrogènes }\end{array}$} \\
\hline $5 \mathrm{mUl}$ & 0 & & \\
\hline $10 \mathrm{mUl}$ & + & Oui & $9 \min$ \\
\hline $20 \mathrm{mUl}$ & + & Oui & $9 \mathrm{~min}$ \\
\hline $100 \mathrm{mUl}$ & + & Oui & $25 \min$ \\
\hline $\begin{array}{c}\text { - brebis cyclique en œestrus } \\
100 \mathrm{mUl}\end{array}$ & + & Oui & $15 \min$ \\
\hline $\begin{array}{l}\text { Adrénaline et noradréna- } 25 \mu \mathrm{g} \\
\text { line (brebis castrée traitée } 50 \mu \mathrm{g} \\
\text { par les cestrogènes) }\end{array}$ & $\begin{array}{l}+ \\
+\end{array}$ & $\begin{array}{l}\text { Oui } \\
\text { Oui }\end{array}$ & $\begin{array}{l}1 \min \\
2 \min \end{array}$ \\
\hline
\end{tabular}

(fig. 5A). Après une injection de $20 \mathrm{mUl}$, la fréquence des salves atteint $6 / \mathrm{min}$ au bout de $3 \mathrm{~min}$; l'augmentation de l'activité dure environ $5 \mathrm{~min}$. Le rythme des salves retourne à sa valeur de base en $8-9 \mathrm{~min}$ (fig. 5A). L'injection de $100 \mathrm{mUl}$ induit une réponse EMG de l'utérus comparable à la réponse de longue durée induite par la dilatation vaginale (fig. $6 \mathrm{~A}$ et $\mathrm{B}$ ). La latence de la réponse est d'une vingtaine de secondes ; la fréquence maximale est atteinte en 1 à 2 min. L'activité du myomètre retrouve son rythme normal en $25 \mathrm{~min}$ (fig. 5A). Plus la dose d'ocytocine injectée est élevée, plus la fréquence des salves et la durée de la réponse augmentent, plus le délai avec lequel est atteint le rythme maximal diminue (fig. 5A). Les potentiels ne sont modifiés ni dans leur amplitude (1 à $2 \mathrm{mV}$ ) ni dans leur fréquence (1 à 1,2 par seconde).

Chez les brebis cycliques, la réponse de l'utérus à une injection de $100 \mathrm{mUI}$ d'ocytocine au cours de l'œstrus persiste $15 \mathrm{~min}$, moins longtemps que chez la Brebis castrée et traitée (fig. 5B).

$2^{\circ}$ Action de l'adrénaline et de la noradrénaline. - Dix secondes après I'injection de $25 \mu \mathrm{g}$ d'adrénaline ou de noradrénaline, chez la brebis castrée sous influence œstrogénique, la fréquence des salves augmente de 1,5 à 5 par minute et la réponse dure une minute.

Si la dose injectée d'adrénaline ou de noradrénaline est augmentée à $50 \mu \mathrm{g}$, la fréquence des salves atteint $6 / \mathrm{min}$. La réponse qui dure $2 \mathrm{~min}$, est comparable à celle de courte durée obtenue après dilatation vaginale (fig. 7A et $\mathrm{B}$ ).

\section{Influence d'un agent $\alpha$-adréno-bloquant.}

$1^{\circ}$ Sur l'activité spontanée et l'activité évoquée par la noradrénaline. - La phentolamine $(5 \mathrm{mg})$ provoque un arrêt de l'activité utérine spontanée, qui dure une à deux minutes. A cette dose, elle bloque l'effet moteur de $50 \mu \mathrm{g}$ de noradrénaline. 

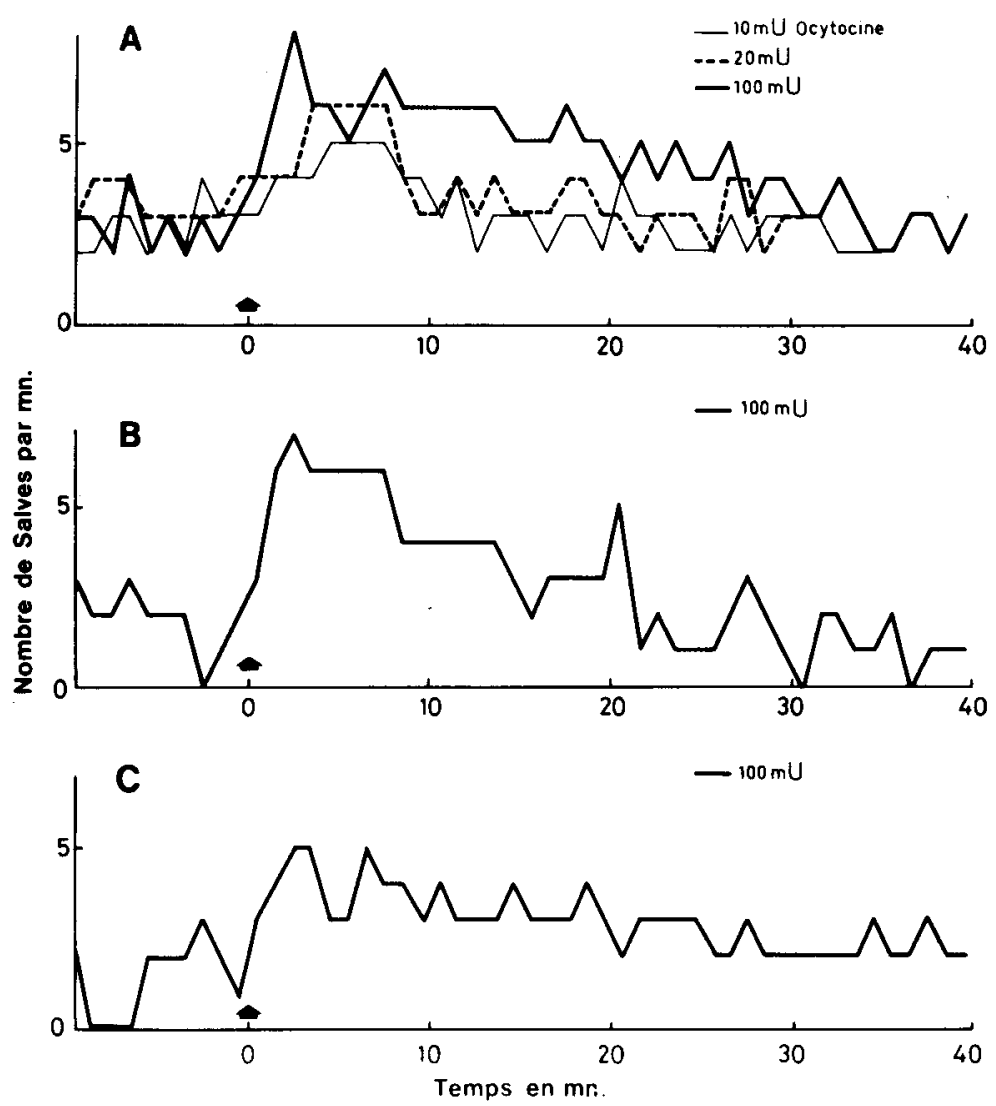

FIG. 5. - Effets d'injections de $10 \mathrm{mU}, 20 \mathrm{mU}, 100 \mathrm{mU}$ d'ocytocine ( $\uparrow$ ) sur la fréquence des salves de potentie/s exprimée en nombre par $10 \mathrm{~min}$.

$A$ : chez une brebis castrée traitée par les cestrogènes ; $B$ : chez une brebis cyclique au cours de I'œstrus: C : chez une brebis castrée, hypophysectomisée, traitée par les œstrogènes. La réponse de l'utérus à une injection de $100 \mathrm{mU}$ d'ocytocine persiste moins longtemps chez la brebis cyclique (B) que chez les brebis castrées traitées ( $\mathrm{A}$ et $\mathrm{C}$ ).

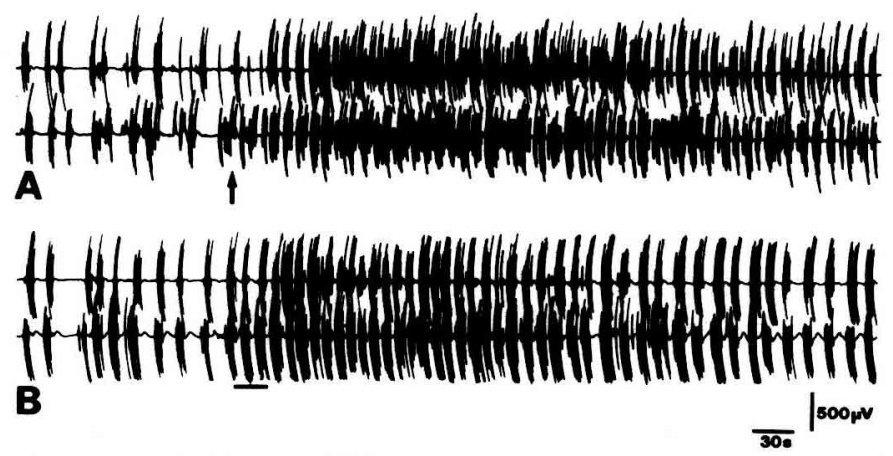

FIG. 6. - Enregistrements de l'activité EMG du myomètre, en deux points d'une même corne chez une brebis castrée traitée par les cestrogènes. L'enregistrement A montre l'augmentation de l'activité EMG en réponse à une injection de $100 \mathrm{mU}$ d'ocytocine (1). En B, la dilatation vaginale (trait horizontal) induit une réponse comparable, de longue durée. 


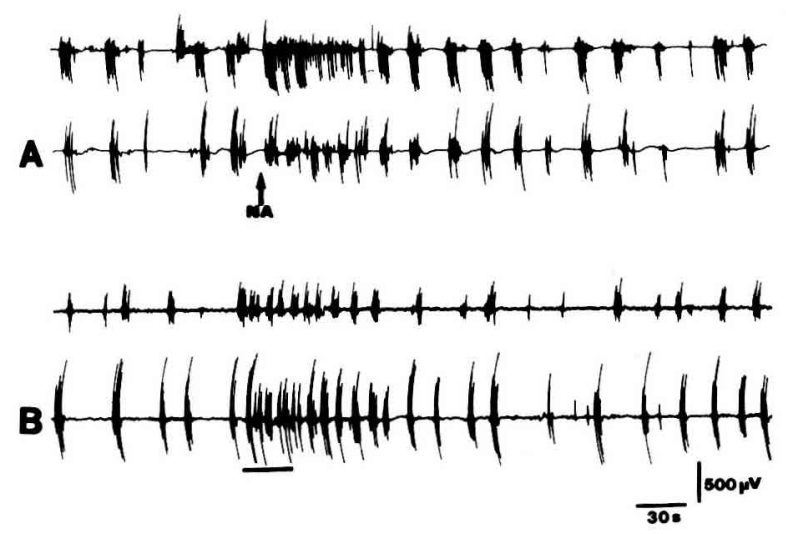

FIG. 7. - Enregistrements de l'activité EMG du myomètre en deux points d'une même corne utérine chez une brebis castrée traitée par les cestrogènes. L'enregistrement A montre l'augmentation de I'activité EMG en réponse à une injection de $50 \mu \mathrm{g}$ de noradrénaline ( 1 ). En $\mathrm{B}$, la dilatation vaginale (trait horizontal) induit une réponse comparable, de courte durée.

$2^{\circ}$ Sur l'activité induite par les stimulations vaginales. - Lorsque la stimulation vaginale consiste en la seule mise en place du spéculum, la phentolamine entraîne une diminution de la réponse utérine. Celle-ci n'est plus qu'une brève salve de potentiels, contemporaine du début de la stimulation (comparer les lig. B et C, fig. 1a). Chez les 3 brebis cycliques en œstrus induit, où la dilatation vaginale produit une augmentation de l'activité EMG du myomètre en deux temps, la phentolamine diminue la bouffée initiale, contemporaine de la stimulation, qui ne dure plus qu'une dizaine de secondes. Par contre, l'augmentation tardive et prolongée du rythme des salves persiste (comparer les lig. $A$ et $B$, fig. $1 b$ ). La phentolamine réduit également les réponses de courte durée à la dilatation vaginale observées chez les brebis castrées sous imprégnation œstrogénique (comparer les lig. $A$ et $B$, fig. 1c).

\section{Influence de l'hypophysectomie.}

$1^{\circ}$ Effet de la dilatation vaginale. - Chez les brebis hypophysectomisées, sous imprégnation œstrogénique, le rythme de base des salves est nettement diminué et l'activité est irrégulière. La réponse à la dilatation vaginale est toujours de courte durée. La fréquence des salves croît de 1 à 5 par minute dès le début de la distension (fig. 8A). L'effet se poursuit tant que dure la stimulation (fig. 8B). Lorsque celle-ci dépasse 3 à $4 \mathrm{~min}$, l'augmentation de l'activité EMG cesse avant la fin de la dilatation vaginale. En présence de phentolamine, la réponse est encore diminuée. II n'est plus recueilli qu'une brève salve, de 6 à $8 \mathrm{~s}$, à l'installation du stimulus (fig. $8 \mathrm{C}$ ).

$2^{\circ}$ Effet de la distension utérine. - Seule la corne distendue par un ballonnet gonflé de $15 \mathrm{ml}$ d'air, présente une modification de l'activité EMG (fig. 4B). La distension fait apparaître, comme chez la brebis témoin, une activité de type " on-off ", à l'installation et à l'arrêt de la stimulation. Au cours du maintien 


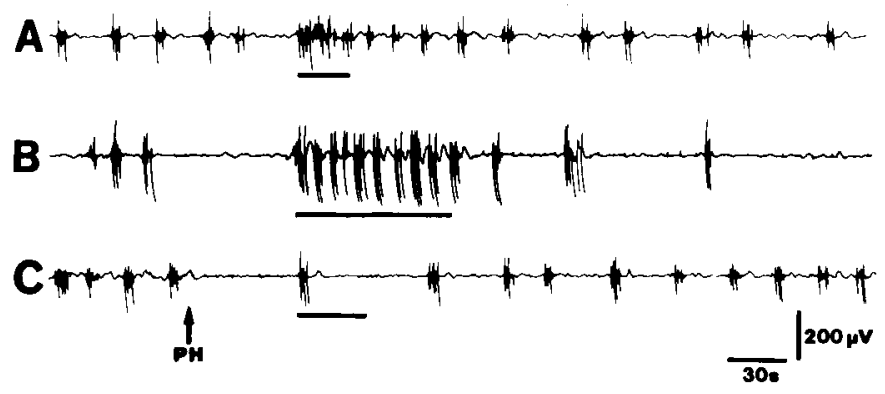

FIG. 8. - Enregistrements de l'activité EMG du myomètre en un point d'une corne utérine, chez une brebis castrée, hypophysectomisée, traitée par les astrogènes. Les traits horizontaux correspondent aux stimulations vaginales.

Les enregistrements $A$ et $B$ montrent l'augmentation de l'activité EMG en réponse à des dilatations vaginales respectivement pendant 30 et $75 \mathrm{~s}$. En C, la réponse à une stimulation identique à celle portée en $A$ est nettement diminuée après une injection de $5 \mathrm{mg}$ de phentolamine (PH). Seule subsiste une brève salve au tout début de la stimulation.

du stimulus, les salves de courte durée ont une fréquence de $5 / \mathrm{min}$. Par rapport au rythme de base, l'augmentation de la fréquence des salves pendant cette période est plus importante chez la brebis hypophysectomisée que chez les brebis témoins. La fréquence des salves recueillies sur la corne contralatérale n'est pas modifiée : elle reste faible, $d$ 'une par minute et irrégulière.

$3^{\circ}$ Action de l'ocytocine. - Chez les animaux hypophysectomisés, I'utérus conserve sa sensibilité à l'ocytocine. L'injection de $100 \mathrm{mUl}$ d'hormone induit après une latence de $25 \mathrm{~s}$, une réponse identique à celle recueillie chez les brebis non hypophysectomisées, en œstrus induit (fig. 5C).

\section{Discussion.}

$1^{\circ}$ Mise en évidence du réflexe de Ferguson chez la Brebis. - La dilatation vaginale détermine une augmentation de l'activité du myomètre chez la plupart des brebis castrées et traitées par les œstrogènes et, dans tous les cas, chez les brebis cycliques. Cette augmentation persiste plus ou moins après l'arrêt de la stimulation. La dilatation du rectum $n^{\prime}$ a par contre aucun effet, contrairement à ce qu'observe Cross (1958) chez la Lapine. L'accélération du rythme des contractions utérines consécutive à la stimulation vaginale chez la brebis n'est pas une manifestation de la réponse de l'organisme à un stress. Elle traduit l'existence d'un réflexe spécifique identique à celui mis en évidence par Ferguson (1941), chez la lapine en post-partum, à la suite de la distension du vagin ou du cervix.

$2^{\circ}$ Mise en évidence des deux composantes de la réponse motrice de l'utérus lors du réflexe. - Pour Ferguson (1941), la nature neuroendocrinienne du réflexe ne fait pas de doute. Pour Cross (1958), il s'agit plutôt d'un réflexe spinal, car la réponse utérine à la distension du vagin est encore présente après la section de la moelle thoracique ou l'hypophysectomie et une anesthésie épidurale la supprime. 
Nous recueillons des réponses à la dilatation vaginale de durée supérieure à 8-10 mn chez 11 des 27 brebis castrées traitées par les œstrogènes et chez toutes les brebis cycliques en œstrus $(n=9)$. Elles ressemblent, par leur durée et la fréquence maximale des salves qui les composent, aux modifications de l'EMG utérine observées 20 secondes après l'injection de $100 \mathrm{mU}$ d'ocytocine. Elles résultent en première analyse de la libération d'ocytocine : la dilatation vaginale entraîne l'augmentation de la concentration plasmatique de cette hormone chez la chèvre (Roberts et Share, 1970 ; Blank et de Bias, 1977) et chez la brebis (Roberts et Share, 1968). En fait, il peut être conclu à l'existence de deux composantes dans ces réponses de longue durée ou de durée intermédiaire. L'hypophysectomie laisse toujours subsister une composante strictement contemporaine de la dilatation vaginale qui, en aucun cas, ne persiste après l'arrêt de la stimulation : la composante tardive, poststimulatoire est supprimée. La sensibilité de l'utérus à l'ocytocine chez les femelles hypophysectomisées étant conservée, nous pouvons conclure que cette composante, qui peut durer pendant 8 à 30 min après l'arrêt de la stimulation chez les brebis intactes, est produite par l'ocytocine. Inversement, la phentolamine déprime la partie de la réponse contemporaine de la dilatation vaginale, mais laisse persister l'augmentation du rythme, bien au-delà de l'arrêt de la stimulation. La première composante de la réponse résulte donc de la mise en jeu d'un réflexe, vraisemblablement spinal, dont les efférences sont des axones sympathiques. La seconde peut être attribuée à l'effet de l'ocytocine, bien que la phentolamine, administrée dans le

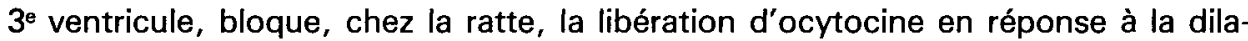
tation vaginale (Moos et Richard, 1975). II est probable que, dans nos expériences, la phentolamine injectée par voie intraveineuse ne franchit pas la barrière hémato-encéphalique et n'atteint pas les neurones hypothalamiques. Les réponses de longue durée à une dilatation de 30 à 40 secondes résulteraient donc de la mise en jeu simultanée d'un réflexe spinal et du réflexe neuroendocrinien dont les effets sont connus pour se manifester après un délai de 35 à $45 \mathrm{~s}$ (Debackere et al., 1961).

Les réponses de courte durée à la dilatation vaginale ou à la seule introduction du spéculum ne sont pas modifiées par l'hypophysectomie. Elles sont déprimées par la phentolamine. II est probable qu'elles résultent de la mise en jeu d'un réflexe plutôt segmentaire, comme le supposait Cross (1958). Le plus souvent, une brève salve de potentiels subsiste, contemporaine du début de la stimulation, chez les brebis hypophysectomisées recevant de la phentolamine : elle traduirait plutôt une réponse locale de l'utérus, peut-être à la stimulation mécanique qu'il subit lors de la mise en place du spéculum.

$3^{\circ}$ Variations saisonnières du réflexe. - L'existence d'une réponse du myomètre à la dilatation vaginale dépend de la saison. Alors qu'en saison sexuelle, nous observons une réponse chez toutes les brebis castrées et traitées par les œstrogènes $(n=16), 5$ brebis sur 11 , soit $45 p$. 100 des animaux, n'en présentent pas pendant la période d'anœstrus, de mars à juillet pour la race Préalpes (Thimonier et Mauléon, 1969). Les éléments nerveux impliqués dans le réflexe de Ferguson sont sensibles aux œstrogènes: ils augmentent le pourcentage des 
neurones du noyau paraventriculaire qui répondent à la dilatation vaginale chez la ratte (Negoro et al., 1973) ; ils potentialisent la libération réflexe d'ocytocine chez la brebis (Roberts et Share, 1969). Comme le comportement d'œestrus chez la brebis castrée est plus difficile à induire par les œstrogènes pendant la période habituelle d'anœstrus que pendant la saison sexuelle (Fletcher et Lindsay, 1971 ; Gibson et Robinson, 1971), il est possible de supposer que le système nerveux central présente des variations saisonnières de sa sensibilité à ces hormones. La diminution du pourcentage des réponses de longue durée, observée pendant l'anœstrus saisonnier chez les brebis castrées et traitées, pourrait s'expliquer par la moindre sensibilité aux œstrogènes des neurones hypothalamiques impliqués dans le réflexe de Ferguson. Comme les réponses courtes sont également moins nombreuses pendant cette période, il faut admettre, dans cette hypothèse; que la diminution de la sensibilité aux œstrogènes affecte aussi les différents éléments périphériques et spinaux du réflexe segmentaire.

$4^{\circ}$ Absence de réflexe à point de départ utérin. - La réponse motrice de l'utérus à sa propre distension n'est vraisemblablement pas de nature réflexe. Si tel était le cas, la distension d'une corne provoquerait une activation de la motricité de l'utérus entier. Or, l'activité de la corne contralatérale à la stimulation n'est jamais modifiée, que les Brebis aient subi ou non I'hypophysectomie. Nos résultats s'opposent à ceux de Ferguson (1941). Pour lui, la distension d'une corne utérine chez la Lapine augmente la motricité de l'autre corne, mais Cross (1958) ne confirme pas ce résultat. La distension utérine ne stimulerait pas de récepteurs comme le fait la dilatation vaginale. Pourtant des récepteurs utérins sensibles à des stimulations mécaniques ont été décrits (Bower, 1959).

L'augmentation de l'activité EMG localisée à la corne stimulée est plutôt due au fait que la fibre musculaire lisse répond à son propre étirement par une dépolarisation de la membrane. Kuriyama (1961) montre qu'au fur et à mesure de l'accroissement de l'étirement, les potentiels d'action naissent d'un potentiel de membrane de plus en plus faible et par conséquent diminuent d'amplitude. $\mathrm{Ce}$ phénomène, étudié au niveau cellulaire, explique vraisemblablement la diminution de l'amplitude des potentiels que nous observons pendant le maintien de la distension. Les salves correspondraient à de petites contractions rythmiques apparaissant sur une activité tonique de base.

Reçu en mai 1981.

Accepté en janvier 1982.

Remerciements. - Nous remercions A. Locatelli (I.N.R.A., Station de Physiologie de la Reproduction, Nouzilly, 37380 Monnaie) pour I'aide efficace qu'il a bien voulu nous apporter en réalisant les hypophysectomies de nos protocoles expérimentaux. Nous remerçions également $\mathrm{G}$. Coutherut pour sa collaboration technique et $\mathrm{M}$. Terriot pour les reproductions photographiques. 


\section{Références}

BLANK M. S., DE BIAS D. A., 1977. Oxytocin release during vaginal distension in the goat. Biol. Reprod., 17, 213-223.

BOWER E. A., 1959. Action potentials from uterine sensory nerves. J. Physiol. Lond., 148, 2P-3P.

CROSS B. A., 1958. The motility and reactivity of the oestrogenized rabbit uterus in vivo; with comparative observation on milk ejection. J. Endocr., 16, 237-260.

DEBACKERE M., PEETERS G., 1960. The influence of vaginal distension on milk ejection and diuresis in the lactating cow. Arch. int. Pharmacodyn. Ther., 123, 462-471.

DEBACKERE M., PEETERS G., TUYTTENS N., 1961. Reflex release of an oxytocic hormone by stimulation of genital organs in male and female sheep studied by a cross circulation technique. J. Endocr., 22, 321-334.

FERGUSON J. K. W., 1941. A study of the motility of the intact uterus at term. Surg. Gynec. Obstet., 73, 359-366.

FLETCHER I. C., LINDSAY D. R., 1971. Effect of oestrogen on oestrous behaviour and its variation with season in the ewe. J. Endocr., 50, 685-696.

GIBSON W. R., ROBINSON T. J., 1971. The seasonal nature of reproductive phenomena in the sheep. I. Variation in sensitivity to oestrogen. J. Reprod. Fert., 24, 9-18.

KURIYAMA H., 1961. Recent studies on the electrophysiology of the uterus, 51-71. In Ciba Foundation Study Group $\mathrm{n}^{\circ}$ 9. Progesterone and the defense mechanism of pregnancy. Churchill Ed., London.

MOOS F., RICHARD P., 1975. Rôle de la noradrénaline et de l'acétylcholine dans la libération d'ocytocine induite par des stimulations vaginale, vagale et mammaire. J. Physiol. (Paris), 70, 315-332.

NEGORO H., VISESSUWAN S., HOLLAND R. C., 1973. Reflex activation of paraventricular nucleus units during the reproductive cycle and in ovariectomized rats treated with oestrogen or progesterone. J. Endocr., 59, 559-567.

PEETERS G., DEBACKERE M., 1963. Influence de la distension du vagin et de la traite ou de la succion sur la diurèse chez la brebis et la chèvre. J. Physiol. (Paris), 55, 481-493.

RICHARD P., 1969. Contribution à l'étude des voies nerveuses du réflexe neuroendocrinien d'éjection du lait chez la brebis. Thèse Fac. Sci., Paris, 172 pp.

ROBERTS J. S., SHARE L., 1968. Oxytocin in plasma of pregnant, lactating and cycling ewes during vaginal stimulation. Endocrinology, 83, 272-278.

ROBERTS J. S., SHARE L., 1969. Effects of progesterone and estrogen on blood levels of oxytocin during vaginal distension. Endocrinology, 84, 1076-1081.

ROBERTS J. S., SHARE L., 1970. Inhibition by progesterone of oxytocin secretion during vaginal stimulation. Endocrinology, 87, 812-815.

ROUSSEAU J. P., PRUD'HOMME M. J., 1974. Etude électromyographique de la motricité de l'utérus chez la brebis. Action des hormones. Ann. Biol. anim. Bioch. Biophys., 14, 67-85.

THIMONIER J., MAULEEON P., 1969. Variations saisonnières du comportement d'œstrus et des activités ovarienne et hypophysaire chez les ovins. Ann. Biol. anim. Bioch. Biophys., 9, 233-250. 